\title{
Resultados da lesão do trato de Lissauer e do corno posterior da medula espinal para o tratamento da dor neuropática central após lesão medular traumática
}

\author{
Luis Roberto Mathias Júnior ${ }^{1}$, Erich Talamoni Fonoff², Manoel Jocobsen Teixeira ${ }^{3}$
}

Divisão de Neurocirurgia do Hospital das Clínicas da Faculdade de Medicina da Universidade de São Paulo, São Paulo, SP, Brasil.

\section{RESUMO}

Realizar uma revisão sobre a eficácia e a segurança da lesão do trato de Lissauer e do corno posterior da medula no tratamento da dor neuropática pós-lesão traumática da medula espinhal. A dor neuropática central tem um grande impacto sobre a qualidade de vida de muitos pacientes com lesão medular traumática. A lesão na zona de entrada da raiz dorsal tornou-se uma das várias opções de tratamento. Apenas estudos primários de pacientes com mais de 13 anos que apresentassem lesão traumática da medula espinhal e dor neuropática central, submetidos à lesão da zona de entrada da raiz dorsal, foram incluídos. Dentre os estudos incluídos, todos eram de série de casos de 5 a 54 pacientes. De acordo com os resultados, pelo menos $50 \%$ dos pacientes atingiram mais de $50 \%$ de alívio da dor ou passaram a apresentar nenhuma limitação dolorosa às atividades diárias habituais, sem a necessidade de opioides. No entanto, todos os estudos analisados apresentavam critérios mal definidos de elegibilidade, ausência de grupo controle e descrição inadequada de efeitos adversos e complicações. Os estudos revisados indicam resultados promissores com a lesão da zona de entrada da raiz dorsal para o tratamento da dor neuropática central em pacientes selecionados com lesão medular traumática. No entanto, o nível de evidências fornecido pelos estudos ainda é baixo, sobretudo em termos do desenho do estudo, avaliação e análise dos resultados, descrição sobre a gravidade dos efeitos adversos e critérios de seleção de pacientes. Por essas razões, apesar dos estudos com resultados favoráveis a essa técnica cirúrgica, o nível de evidência científica permanece fraco.

\section{PALAVRAS-CHAVE}

Dor, ondas de rádio, dor intratável, coagulação por laser, traumatismos da medula espinal.

\section{ABSTRACT}

Results of dorsal root entry zone (DREZ) lesioning for central neuropathic pain due to traumatic spinal cord injuries

To review the efficacy and safety of the Lissauer's tract and the dorsal horn of the spinal cord lesion in the treatment of neuropathic pain after traumatic spinal cord injury. Central neuropathic pain has a major impact on the quality of life for many patients with traumatic spinal cord injury. Lesion in the dorsal root entry zone has become one of the various treatment options. Only primary studies of patients with more than 13 years old, who presented traumatic spinal cord injury and central neuropathic pain, receiving dorsal root entry zone lesioning were included. Among the included studies, all were case series ranging from 5 to 54 patients. According to the results, at least $50 \%$ of patients achieved more than $50 \%$ of pain relief or experienced no pain limitation for activity, without the need for opioids. However, all studies analyzed had poorly defined eligibility criteria, lack of control groups and inadequate description of adverse effects and complications. The studies reviewed indicate promising results with the dorsal root entry zone lesioning for the treatment of central neuropathic pain in selected patients with traumatic spinal cord injury. However, the level of evidence provided by the studies is still low, especially in terms of study design, evaluation and analysis of results, description of the severity of adverse effects and patient selection criteria. For these reasons, although there are studies with favorable results with this surgical technique, the level of scientific evidence remains weak.

\section{KEYWORDS}

Pain, radio waves, intractable pain, laser coagulation, spinal cord injuries.

1 Médico-residente da Divisão de Clínica Neurocirúrgica do Hospital das Clínicas da Faculdade de Medicina da Universidade de São Paulo (HC-FMUSP), São Paulo, SP, Brasil.

2 Coordenador do Grupo de Neurocirurgia Funcional do HC-FMUSP, São Paulo, SP, Brasil.

3 Professor titular de Neurocirurgia da Faculdade de Medicina da USP; diretor da Divisão de Clínica Neurocirúrgica do HC-FMUSP, São Paulo, SP, Brasil. 


\section{Introdução}

A dor tem sido reconhecida por mais de meio século como um dos muitos sintomas vivenciados por pacientes que apresentam lesão medular, sobretudo em plexo braquial. ${ }^{1-5}$ A prevalência, a gravidade e a duração da dor nessa população de pacientes, no entanto, apresentam grande variação. Estudos descrevem que a dor é vivenciada desde $1 \%$ até $94 \%$ das pessoas em algum momento depois de uma lesão medular ${ }^{6-8}$ e pode se tornar crônica em $1 \%$ a $70 \%$ dos casos. Em $5 \%$ a $37 \%$ dessa população, a dor pode ser muito grave ou incapacitante..$^{9-11}$ Essa variação nas estimativas de prevalência, gravidade e duração da dor tem sido explicada por diferenças entre os estudos no que se refere à definição de dor, terminologia, classificação, critérios de inclusão e variabilidade na metodologia, bem como entre diversos fatores etiológicos, demográficos e culturais. ${ }^{7}$

Em 1997, Siddall et al. ${ }^{8}$ desenvolveram uma classificação para dor que vem ganhando aceitação, já que se baseou na literatura existente e foi projetada para fornecer orientações simples tanto para pesquisa quanto para definição de conduta.

O primeiro eixo dessa classificação inclui quatro grandes tipos ou divisões da dor: lesões musculoesqueléticas, visceral, neuropática e outras. ${ }^{8}$ Essa divisão é baseada no sistema afetado, que pode ser prontamente identificado apenas com a avaliação clínica. Nesta revisão a dor neuropática é que está em foco e, portanto, será a única discutida.

A dor neuropática pode ocorrer após lesão ao sistema nervoso central ou periférico. ${ }^{12}$ Ela pode ser identificada por meio das características da dor (choque, queimor, pontada) ou pela área de acometimento (região de perturbação sensorial), que pode ser subdividida em dor neuropática "ao nível” ou "abaixo do nível" da lesão. ${ }^{8}$

A dor "ao nível” ocorre no nível correspondente da lesão medular em um padrão segmentar com características neuropáticas e inclui dois segmentos acima e dois abaixo do nível da lesão medular, uma vez que a aferência de vários segmentos pode estar interrompida ou alterada após a agressão em qualquer nível em particular. Essa dor é descrita como "queimor", "formigamento", "pontada" ou "tiro", em uma distribuição de dermátomo ao nível da lesão, com ou sem hiperestesia. Esse tipo de dor pode ser atribuído à patologia da raiz nervosa, a alterações na medula espinhal ou em estruturas supraespinhais. ${ }^{8}$

Dor neuropática abaixo do nível da lesão refere-se a uma dor difusa, que também é descrita com as palavras "queimor", "área dolorida", "sensação de tiro" ou de "punhalada”. Diferentemente da dor "ao nível", essa dor ocorre pelo menos três níveis abaixo da lesão. ${ }^{8}$ Este tipo de dor tem sido denominada por outros sistemas de classificação como "dor central” ou "dor por desaferentação" ${ }^{13}$ Nesta revisão, a dor neuropática central (DNC) refere-se tanto àquela ao nível da lesão como à dor neuropática abaixo do nível da lesão, resultantes da patologia da medula espinhal ou de estruturas supraespinhais.

As estimativas da prevalência DNC obtidas a partir de amostras tomadas na Suécia, no Reino Unido e nos Estados Unidos variam de $42 \%$ a $69 \% .{ }^{14-17}$ Prevalência em outros estudos relatam estimativas que variam de $10 \%$ a $70 \%$, embora a maioria dos estudos relate estimativas entre $40 \%$ e $70 \% .^{10,14,18-30}$

No geral, esses estudos mostram que a DNC inicia-se logo após a lesão medular traumática (LMT), mantendo-se como um problema persistente, mesmo anos após a agressão inicial. A ampla variação nas taxas de prevalência pode ser atribuída a vários problemas metodológicos, tais como a falta de uma definição uniforme para DNC, técnicas inadequadas de amostragem e tempo variável entre o momento da lesão até a avaliação da dor.

Embora haja heterogeneidade nas taxas de prevalência, DNC é um problema significativo após LMT. Inclusive, a dor pode exercer um impacto ainda maior sobre a qualidade de vida do paciente do que a limitação motora e funcional provocada pela própria lesão.

Em um esforço para controlar e melhorar a qualidade de vida dos pacientes acometidos, procedimentos cirúrgicos foram idealizados e desenvolvidos. A lesão do trato de Lissauer e do corno posterior da medula espinhal (DREZ - dorsal root entry zone) é um procedimento cirúrgico destinado à ablação dos neurônios dessa região que demonstram hiperatividade paroxística após lesão de desaferentação ${ }^{8}$ (Figura 1). Em 1942, uma tratotomia de Lissauer, a primeira abordagem cirúrgica atuando na zona de entrada da raiz dorsal em humanos, foi realizada objetivando o alívio da dor. ${ }^{31}$ Ainda na tentativa de aprimorar a técnica, em 1966 foi realizado em gatos o experimento denominado de ablação por radiofrequência da zona de entrada da raiz dorsal (DREZ) ${ }^{32}$ Posteriormente, o procedimento foi refinado e, em 1979, Nashold e Ostahl ${ }^{33}$ realizam pela primeira vez uma microcoagulação da DREZ para o tratamento de dor pós-avulsão do plexo braquial (Figura 2). Em 1981, Nashold e Bullitt ${ }^{34}$ usaram o mesmo procedimento para o tratamento da dor por desaferentação pós-traumatismo raquimedular.

Rawlings et al. ${ }^{35}$ indicaram que as camadas Rexed I a V são a origem do trato espinotalâmico, bem como a região-alvo do procedimento ablativo. Após a desaferentação, há indícios de hiperatividade neuronal e mudança na concentração relativa de substância $\mathrm{P}$ e betaendorfinas nessas camadas. $\mathrm{O}$ alívio da dor após a intervenção cirúrgica pode decorrer de três dos seguintes mecanismos: ${ }^{36}$

- interrupção das vias ascendentes de dor das colunas dorsais e dorsolaterais; 


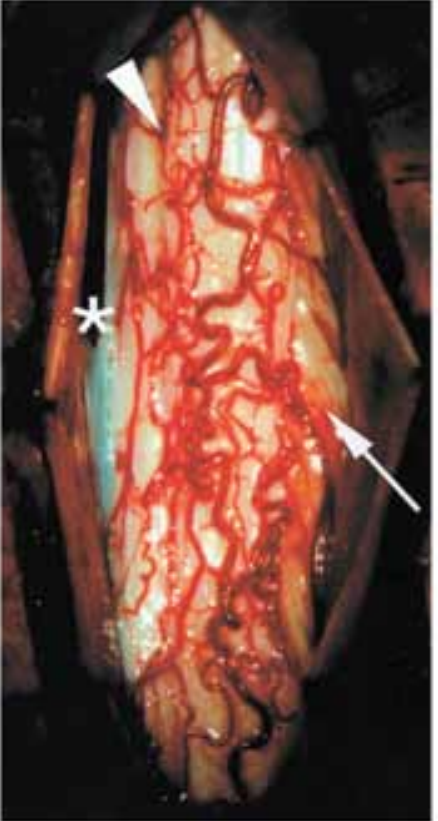

Figura 1 - Fotografia intraoperatória da porção dorsal da medula espinhal. O lado intacto da superfície medular mostra raízes dorsais e vasos (seta), em oposição ao lado avulsionado, com ausência de raízes dorsais e formação cicatricial ( $\left.{ }^{*}\right)$, aderência dural e rarefação de vasos sanguíneos. O sulco intermédio-lateral, com ausência de raízes dorsais é indicado (seta).

- destruição dos centros geradores de dor na medula;

- reequilíbrio dos estímulos inibitórios e excitatórios da rede sensorial danificada.

A lesão na zona de entrada da raiz dorsal pode ser feita utilizando-se radiofrequência, microcoagulação com bisturi bipolar, laser ou lâmina cortante de bisturi. ${ }^{37}$ Recentemente, a assistência por computador tem sido incorporada à ablação por radiofrequência para melhorar a precisão da lesão. ${ }^{38} \mathrm{Com}$ o avanço da tecnologia, ablação com laser foi desenvolvida para produzir menores e mais discretas lesões. ${ }^{37}$ Tipos específicos de laser incluem o dióxido de carbono (CO2), argônio e neodímio: ítrio alumínio (Nd: YAG).

\section{Eficácia da lesão da zona de entrada}

Em três estudos em que foi utilizada a radiofrequência, ${ }^{39,40}$ os autores relataram que houve bom alívio da dor em 26 (48\%) dos 54 pacientes, 12 (75\%) dos 16 pacientes e 12 (41\%) dos 29 pacientes com lesão traumática da medula espinhal, um ano após a cirurgia, imediatamente após a cirurgia e durante um período médio de acompa-

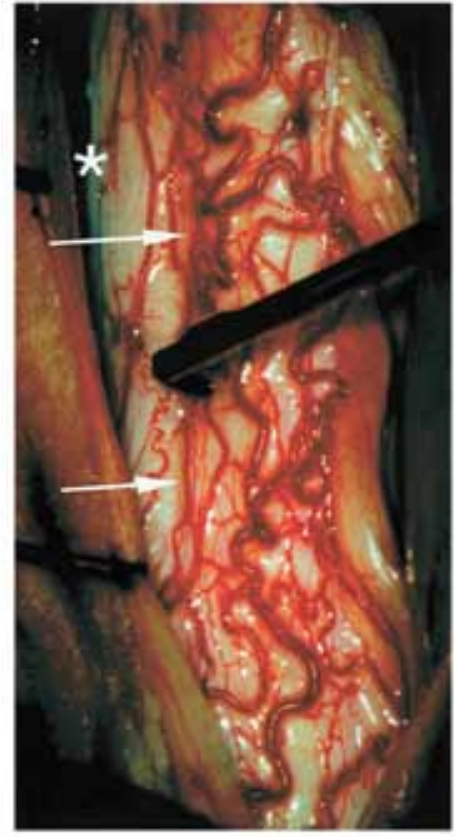

Figura 2 - Fotografia intraoperatória indicando o ângulo recomendado de introdução do eletrodo de radiofrequência. Inserção do eletrodo no sulco intermédio-lateral, em um ângulo de 30 graus em relação à linha sagital (setas). O eletrodo é inserido na medula espinhal a uma profundidade de $2 \mathrm{~mm}$; objetiva-se uma densidade de 1 lesão/mm. A falta de raízes dorsais no lado lesado é indicada $\left(^{\star}\right)$.

nhamento de três anos após a cirurgia, respectivamente. Bom alívio da dor foi definido como ausência de uso de analgésicos e nenhuma limitação das atividades habituais devido à dor. ${ }^{39,41}$ Nesses mesmos estudos, os autores também relataram que $3(6 \%)$ dos 54 pacientes, 4 (25\%) dos 16 pacientes e 7 (24\%) dos 29 pacientes apresentaram alívio moderado da dor (ausência de uso de opioides e sem limitação de atividades habituais pela dor) durante o mesmo período de segmento.

Em dois outros estudos envolvendo o uso da radiofrequência, o "primeiro" ${ }^{41}$ relatou que 4 (67\%) de 6 pacientes apresentaram alívio de $100 \%$ da dor durante um período médio de 9,1 meses de acompanhamento, enquanto o "segundo" 42 informou que 8 (53\%) dos 15 pacientes apresentaram alívio de $100 \%$ da dor por um período de tempo não descrito. Além disso, os dois pacientes restantes do "primeiro" estudo anterior alcançaram $80 \%$ de alívio da dor durante o mesmo período de segmento.

Em outros dois estudos utilizando radiofrequência, ${ }^{34,43}$ os autores relataram mais de $50 \%$ de alívio da dor em $7(78 \%)$ dos 9 pacientes por um período que variou de 5 a 38 meses e em 4 (80\%) de 5 pacientes com LMT, 2-3 dias após a cirurgia. Finalmente, em um estudo semelhante, ${ }^{44}$ os autores relataram que 11 (48\%) dos 23 pacientes com LMT apresentaram mais de $75 \%$ de alívio da dor em uma média de 51 meses após realizarem ablação da "DREZ" por radiofrequência. 
No único estudo utilizando assistência computadorizada para aumentar a acurácia de identificação da zona de entrada, ${ }^{38}$ os autores relataram que $84 \%$ dos pacientes com LMT apresentaram $100 \%$ de alívio da dor (não especificando o período de melhora), enquanto $92 \%$ apresentaram alívio da dor entre 50\%-100\% durante um período de seguimento médio de 44 meses.

No único estudo relatando a eficácia da ablação por meio de radiofrequência e laser de $\mathrm{CO} 2$, foi obtido alívio satisfatório da dor (definida como uma redução na intensidade de dor em pelo menos 50\%, interrupção do uso de opioides e melhora da capacidade funcional) em $8(57 \%)$ dos 14 pacientes com LMT submetidos à radiofrequência e 3 (50\%) de 6 pacientes submetidos a laser. ${ }^{45}$

Finalmente, no estudo sobre o uso de três diferentes tipos de laser (CO2, argônio, Nd: YAG), os resultados foram apresentados agrupando os resultados dos três tipos de laser. ${ }^{37}$ Os autores relataram que 5 (56\%) de 9 pacientes com LMT apresentaram alívio da dor superior a $50 \%$, não necessitando de uso de opioides durante um período de seguimento médio de 24 meses.

\section{Efeitos adversos}

Em um estudo sobre o uso de radiofrequência como técnica ablativa, não houve ocorrência de infecção, fístula liquórica ou déficit motor. ${ }^{41}$ Efeito adverso comum em quatro estudos foi a ocorrência de déficit motor logo após o procedimento cirúrgico, observado em 14 $(23 \%)$ de 61 pacientes. Ocorrência de fístula liquórica e infecção da ferida foi descrito por dois estudos, ${ }^{46} \mathrm{com}$ incidência similar de ambas as complicações em 3 (9\%) de 32 pacientes. Apesar da diversidade de efeitos adversos possíveis, déficits motor e sensorial permaneceram como os mais comumente relatados. ${ }^{38,39}$

\section{Discussão}

A partir da revisão apresentada, estudos indicam que a lesão do corno posterior da medula e do trato de Lissauer no tratamento da DNC oferece resultados promissores. Essa intervenção mostra resultados favoráveis em termos de alívio da dor para mais da metade dos pacientes. No entanto, esses resultados para DNC devem ser interpretados com cautela. A ablação do corno posterior da medula e do trato de Lissauer também tem sido utilizada com sucesso no tratamento da dor neuropática resultante de avulsão radicular. ${ }^{33,47,48}$ Dados a natureza e o mecanismo das lesões traumáticas, é possível que alguns dos pacientes descritos como tendo patologia central pudessem apresentar lesões periféricas (ou seja, lesão por avulsão) concomitantes. Assim, embora esta revisão tenha assumido como critério de inclusão pacientes com patologias centrais, os resultados positivos aqui descritos podem também refletir a redução bem-sucedida da dor neuropática resultante de patologia periférica associada.

Também é importante salientar que a força de evidência fornecida pelos estudos revisados é de baixo impacto. Trata-se de estudos observacionais, não randomizados, com ausência de grupo controle. Essas limitações aumentam as variáveis de confusão e potenciais resultados inválidos. Por essa razão, permanece incerto se a melhoria na DNC desses pacientes deve ser atribuída ao acaso, ao efeito placebo, à evolução natural desse tipo de dor ou à eficácia do procedimento cirúrgico.

Além disso, as descrições e detalhes sobre os parâmetros utilizados nos procedimentos e locais exatos da intervenção cirúrgica foram insuficientes. Rawlings et al. ${ }^{35}$ sugeriram que os parâmetros de técnica cirúrgica são importantes, e Sindou et al. ${ }^{49}$ propuseram que a ablação abaixo do local da lesão é ineficaz. Portanto, generalização dos resultados desses estudos a outros grupos de pacientes com LMT que apresentam DNC possui limitação para exata reprodutibilidade.

\section{Conclusão}

Mesmo com problemas de validade e generalização dos estudos, a cirurgia ablativa do corno posterior da medula espinhal e do trato de Lissauer assume importante papel como último recurso quando outras intervenções paliativas não apresentarem êxito.

\section{Referências}

1. Anke AG, Stenehjem AE, Stanghelle JK. Pain and life quality within 2 years of spinal cord injury. Paraplegia. 1995;33(10):555-9.

2. Backonja MM, Galer BS. Pain assessment and evaluation of patients who have neuropathic pain. Neurol Clin. 1998;16(4):775-90.

3. Bedbrook G. Pain and phantom sensation. In: Bedbrook $\mathrm{G}$, editor. The care and management of spinal cord injuries. New York: Springer Verlag; 1981. p. 224-9.

4. Berić A, Dimitrijević MR, Lindblom U. Central dysesthesia syndrome in spinal cord injury patients. Pain. 1988;34(2): 109-16.

5. Botterell EH, Callaghan JC, Jousse AT. Pain in paraplegia; clinical management and surgical treatment. Proc R Soc Med. 1954;47(4):281-8. 
6. Rath SA, Braun V, Soliman N, Antoniadis G, Richter HP. Results of DREZ coagulations for pain related to plexus lesions, spinal cord injuries and postherpetic neuralgia. Acta Neurochir (Wien). 1996;138(4):364-9.

7. Roth EJ. Pain in spinal cord injury. In: Yarkony G, editor. Spinal cord injury: medical and rehabilitation management. Gaithersburg: Aspen Publishers; 1994. p. 141-58.

8. Siddall PJ, Taylor DA, Cousins MJ. Classification of pain following spinal cord injury. Spinal Cord. 1997;35(2):69-75.

9. Kaplan LI, Grynbaum BB, Lloyd KE, Rusk HA. Pain and spasticity in patients with spinal cord dysfunction. Results of a follow-up study. JAMA. 1962;182:918-25.

10. Nepomuceno C, Fine PR, Richards JS, Gowens H, Stover $\mathrm{SL}$, Rantanuabol U, et al. Pain in patients with spinal cord injury. Arch Phys Med Rehabil. 1979;60(12):605-9.

11. Ravenscroft A, Ahmed YS, Burnside IG. Chronic pain after spinal cord injury: a survey of practice in UK spinal injury units. Spinal Cord. 1999;37(1):25-8.

12. Merskey H, Bogduk N. Classification of chronic pain. Seattle: IASP Press; 1994.

13. Donovan WH, Dimitrijevic MR, Dahm L, Dimitrijevic M. Neurophysiological approaches to chronic pain following spinal cord injury. Paraplegia. 1982;20(3):135-46.

14. Levi R, Hultling C, Seiger A. The Stockholm Spinal Cord Injury Study: 2. Associations between clinical patient characteristics and post-acute medical problems. Paraplegia. 1995;33(10):585-94.

15. Rintala DH, Loubser PG, Castro J, Hart KA, Fuhrer MJ. Chronic pain in a community-based sample of men with spinal cord injury: prevalence, severity, and relationship with impairment, disability, handicap, and subjective well-being. Arch Phys Med Rehabil. 1998;79(6):604-14.

16. Rose M, Robinson JE, Ells P, Cole JD. Pain following spinal cord injury: results from a postal survey. Pain. 1988;34(1):101-2.

17. Turner JA, Cardenas DD. Chronic pain problems in individuals with spinal cord injuries. Semin Clin Neuropsychiatry. 1999;4(3):186-94.

18. Burke DC. Pain in paraplegia. Paraplegia. 1973;10(4): 297-313.

19. Cairns DM, Adkins RH, Scott MD. Pain and depression in acute traumatic spinal cord injury: origins of chronic problematic pain? Arch Phys Med Rehabil. 1996;77(4): 329-35.

20. Cobeljic R, Kovacevic S, Vulovic D. Types of chronic pain in patients with spinal cord injury. Acta Orthop Yugosl. 1998;29(1):73-7.

21. Demirel G, YIlımaz H, Gençosmanoğlu B, Kesiktaş N. Pain following spinal cord injury. Spinal Cord. 1998;36(1):25-8.

22. Fenollosa P, Pallares J, Cervera J, Pelegrin F, Inigo V, Giner $\mathrm{M}$, et al. Chronic pain in the spinal cord injured: statistical approach and pharmacological treatment. Paraplegia. 1993;31(11):722-9.

23. New PW, Lim TC, Hill ST, Brown DJ. A survey of pain during rehabilitation after acute spinal cord injury. Spinal Cord. 1997;35(10):658-63.

24. Richards JS, Meredith RL, Nepomuceno C, Fine PR, Bennett G. Psycho-social aspects of chronic pain in spinal cord injury. Pain. 1980;8(3):355-66.

25. Richards JS, Stover SL, Jaworski T. Effect of bullet removal on subsequent pain in persons with spinal cord injury secondary to gunshot wound. J Neurosurg. 1990;73(3): 401-4.

26. Siddall PJ, Taylor DA, McClelland JM, Rutkowski SB, Cousins MJ. Pain report and the relationship of pain to physical factors in the first 6 months following spinal cord injury. Pain. 1999;81(1-2):187-97.

27. Störmer S, Gerner HJ, Grüninger W, Metzmacher K, Föllinger S, Wienke C, et al. Chronic pain/dysaesthesiae in spinal cord injury patients: results of a multicentre study. Spinal Cord. 1997;35(7):446-55.
28. Sved P, Siddall PJ, McClelland J, Cousins MJ. Relationship between surgery and pain following spinal cord injury. Spinal Cord. 1997;35(8):526-30.

29. Wang D, Bodley R, Sett P, Gardner B, Frankel H. A clinical magnetic resonance imaging study of the traumatised spinal cord more than 20 years following injury. Paraplegia. 1996;34(2):65-81.

30. Woolsey RM. Chronic pain following spinal cord injury. $J$ Am Paraplegia Soc. 1986;9(3-4):39-41.

31. Hyndman OR. Lissauer's tract section: a contribution to chordotomy for the relief of pain (preliminary report). J Int Coll Surg. 1942;5:294-400.

32. Yaksh TL, Frederick WL. Kerr: comments on his life with notes on his contributions to the understanding of facial neuralgias. Appl Neurophysiol. 1988;51(2-5):65-75.

33. Nashold BS Jr, Ostdahl RH. Dorsal root entry zone lesions for pain relief. J Neurosurg. 1979;51(1):59-69.

34. Nashold BS Jr, Bullitt E. Dorsal root entry zone lesions to control central pain in paraplegics. J Neurosurg. 1981;55(3):414-9.

35. Rawlings CE 3rd, el-Naggar AO, Nashold BS Jr. The DREZ procedure: an update on technique. $\mathrm{Br} \mathrm{J}$ Neurosurg. 1989;3(6):633-42.

36. Richter HP, Seitz K. Dorsal root entry zone lesions for the control of deafferentation pain: experiences in ten patients. Neurosurgery. 1984;15(6):956-9.

37. Powers SK, Barbaro NM, Levy RM. Pain control with laserproduced dorsal root entry zone lesions. Appl Neurophysiol. 1988;51(2-5):243-54.

38. Edgar RE, Best LG, Quail PA, Obert AD. Computerassisted DREZ microcoagulation: posttraumatic spinal deafferentation pain. J Spinal Disord. 1993;6(1):48-56.

39. Friedman AH, Nashold BS Jr. DREZ lesions for relief of pain related to spinal cord injury. J Neurosurg. 1986;65(4):465-9.

40. Nashold BS Jr, Vieira J, el-Naggar AO. Pain and spinal cysts in paraplegia: treatment by drainage and DREZ operation. Br J Neurosurg. 1990;4(4):327-35.

41. Spaić M, Petković S, Tadić R, Minić L. DREZ surgery on conus medullaris (after failed implantation of vascular omental graft) for treating chronic pain due to spine (gunshot) injuries. Acta Neurochir (Wien). 1999;141(12):1309-12.

42. Wiegand $\mathrm{H}$, Winkelmüller $\mathrm{W}$. [Treatment of deafferentation pain by high-frequency intervention on the dorsal root entry zone]. Dtsch Med Wochenschr. 1985;110(6):216-20.

43. Samii M, Moringlane JR. Thermocoagulation of the dorsal root entry zone for the treatment of intractable pain. Neurosurgery. 1984;15(6):953-5.

44. Rath SA, Seitz K, Soliman N, Kahamba JF, Antoniadis G, Richter HP. DREZ coagulations for deafferentation pain related to spinal and peripheral nerve lesions: indication and results of 79 consecutive procedures. Stereotact Funct Neurosurg. 1997;68(1-4 Pt 1):161-7.

45. Young RF. Clinical experience with radiofrequency and laser DREZ lesions. J Neurosurg. 1990;72(5):715-20.

46. Sampson JH, Cashman RE, Nashold BS Jr, Friedman $\mathrm{AH}$. Dorsal root entry zone lesions for intractable pain after trauma to the conus medullaris and cauda equina. $J$ Neurosurg. 1995;82(1):28-34.

47. Campbell JN, Solomon CT, James CS. The Hopkins experience with lesions of the dorsal horn (Nashold's operation) for pain from avulsion of the brachial plexus. Appl Neurophysiol. 1988;51(2-5):170-4.

48. Thomas DG, Kitchen ND. Long-term follow up of dorsal root entry zone lesions in brachial plexus avulsion. $J$ Neurol Neurosurg Psychiatry. 1994;57(6):737-8.

49. Sindou M, Mertens P, Garcia-Larrea L. Surgical procedures for neuropathic pain. Neurosurg Q. 2001;11:45-65.

Endereço para correspondência

Rua Alves Guimarães, 518, ap. 135, Pinheiros

05410-000 - São Paulo, SP

Telefone: (11) 8964-3114

E-mail: lrmathias_jr@hotmail.com 